\title{
Realimentação de Força para Teleoperação de Escavadeiras *
}

\author{
Matheus V. de Lima* André Cid ${ }^{* * *}$ Felipe Cadar* \\ Luan Pinto* Paulo Rezeck* Thiago Grabe* \\ Gustavo Freitas ${ }^{* *, * * *}$ Douglas G. Macharet* \\ Gustavo Pessin ${ }^{* * *}$ Luiz Chaimowicz * Mario F. M. Campos* \\ * Departamento de Ciência da Computação, Universidade Federal de \\ Minas Gerais (UFMG), MG, (e-mail: \{matheusvl, luang\}@ufmg.br, \\ \{cadar, rezeck, thiago.grabe, doug, chaimo, mario\}@dcc.ufmg.br). \\ ** Departamento de Engenharia Elétrica, Universidade Federal de \\ Minas Gerais (UFMG), MG (e-mail: gustavomfreitas@ufmg.br) \\ *** Intituto Tecnológico da Vale, MG (e-mail: andre.cid@pq.itv.org, \\ gustavo.pessin@itv.org)
}

\begin{abstract}
Lately, we have witnessed a growing effort to improve the working conditions by adopting measures to increase overall safety. In this context, teleoperation appears to improve human capacities during remote equipment operations. Teleoperation systems must provide the operator with necessary information from the remote equipment and environment to enable efficient task performance. The mining industry is an area where the use of teleoperation can be advantageous. For example, the remote operation of heavy-duty equipment such as excavators may bring improvements in safety and efficiency. This paper aims to describe an effective approach to provide force feedback in the context of a framework for excavator teleoperation. The methodology is evaluated through loading tasks simulations. The results show that the force feedback can improve the task accomplishment by providing immersion to the operator, including the sense of the load in the bucket and interaction forces with obstacles - which can lead to significant reductions in the stress applied to the manipulator.

Resumo: É crescente o interesse em ambientes com melhores condições de trabalho, evitando locais insalubres e valorizando a qualidade de vida dos trabalhadores. Nesse contexto, a teleoperação aparece para melhorar as capacidades humanas na manipulação de equipamentos remotos. Para uma melhor realização da tarefa, devem ser fornecidas ao operador as informações necessárias para tornar a interação com o ambiente remoto a mais imersiva possível. A indústria de mineração é uma área em que a teleoperação pode ser aplicada, por exemplo, para teleoperar equipamentos como escavadeiras, obtendo melhorias em segurança e eficiência. Este artigo tem como objetivo o desenvolvimento de um arcabouço para a teleoperação de escavadeiras com realimentação de força. A metodologia foi avaliada através da simulação de tarefas de carregamento. Os resultados mostram que a realimentação de força pode melhorar a realização da tarefa ao promover uma imersão ao operador, incluindo sensações de carga carregada na caçamba e de forças de interação com obstáculos - esta informação pode levar a uma significante redução do estresse ao qual o manipulador é submetido.
\end{abstract}

Keywords: Teleoperation; Force feedback; Robotic Manipulator; Haptic Device; Mining Equipment.

Palavras-chaves: Teleoperação; Realimentação de Força; Manipulador; Dispositivo Háptico; Equipamentos de Mineração.

\section{INTRODUÇÃO}

Mineração é uma importante indústria extrativa, em escala global, que nos últimos anos tem recebido grande impulso pela utilização de técnicas de automação e robótica. Um dos principais objetivos dessa utilização consiste na redu-

\footnotetext{
* Este trabalho foi parcialmente financiado pelo Conselho Nacional de Desenvolvimento Científico e Tecnológico - Brasil (CNPq), pelo Instituto Tecnológico da Vale (ITV), pela Embrapii (DCC-UFMG) e pela Fundação de Amparo à Pesquisa de Minas Gerais (FAPEMIG).
}

ção da exposição desnecessária de pessoas à situações de risco ou insalubres. Nesse contexto, a automação já provou ser um meio para um ganho em segurança e produtividade (Roberts et al., 2002).

Um passo em direção à automação completa de uma atividade em mineração é a teleoperação, cujo objetivo é estender as capacidades humanas para manipular objetos remotos e operar equipamentos em ambientes perigosos (Lichiardopol, 2007). Devido a restrições físicas, tais como a localização geográfica do operador e do ambiente remoto, 
há pelo menos dois elementos envolvidos na teleoperação: o mestre/local e o escravo/remoto (Niemeyer et al., 2016). O ambiente local inclui o operador humano e todos os equipamentos e dispositivos necessários para a teleoperação, como joystick, teclados e monitores. $\mathrm{O}$ ambiente remoto contém o equipamento robótico, os sensores e atuadores responsáveis pela interação com o ambiente e realização da tarefa (Hokayem e Spong, 2006).

Uma forma de melhorar o desempenho em tarefas de teleoperação é a utilização de realimentação háptica. O termo "háptico" vem do grego "haptikos" que significa aquilo que está relacionado ao sentido do tato. Na literatura de robótica e de realidade virtual, háptica é definida como interações de forças e torques entre humanos, robôs e ambientes (remotos ou simulados), em suas várias combinações (Park e Khatib, 2006). O objetivo da tecnologia háptica é prover ao usuário a percepção mais fidedigna possível de estar fisicamente manipulando o objeto ou ambiente remoto (Okamura, 2009).

Se o dispositivo escravo (remoto) possuir sensor de força/torque em seu efetuador ou sensores de força/torque em suas juntas, torna-se possível a transmissão dessas forças/torques de interação para o operador durante a realização da tarefa. Essa informação é transmitida ao operador por meio de uma interface háptica, que é capaz de receber e transmitir dados de força e torque para o usuário. Além da informação visual, o envio para o usuário da força de contato do dispositivo robótico (remoto) ocasionado pela interação com o ambiente é importante para a realização da tarefa. Durante operações de escavação, é comum o surgimento de cortinas de poeira que prejudicam a realimentação visual, o que pode ser mitigado pela informação de força da interação. Por exemplo, uma vez que tal interface possibilita ao operador sentir a força de contato remoto e ter a noção da carga sendo depositada na caçamba, parte do problema da falta de informação visual é contornada. Outros benefícios incluem o aumento da segurança e vida útil do equipamento e, por consequência, a redução de custos de manutenção advindos da redução do estresse ao qual o manipulador é submetido (Ahn, 2004). O aumento da vida útil é devido ao maior cuidado que o operador tem quando tem a realimentação de força, não expondo assim o manipulador a forças de interação excessivas.

Existem duas arquiteturas básicas de funcionamento para os dispositivos local e remoto: posição-posição e posiçãoforça. Em arquiteturas do tipo posição-força, obtém-se a força exercida no efetuador do manipulador remoto, e então esta força é realimentada através do dispositivo háptico. Isto possibilita ao operador sentir as forças de interação entre o robô remoto e o seu ambiente. Entretanto, esta arquitetura é menos estável e pode apresentar um alto ganho na realimentação de força; isto significa, na prática, que um pequeno comando de movimento pode virar uma grande força se o remoto estiver pressionado contra um ambiente rígido (Niemeyer et al., 2016).

Este artigo apresenta uma estrutura mínima para teleoperação de um manipulador que emula um braço de escavadeira, na qual o operador pode atuar no ambiente remoto/virtual por meio de uma interface háptica que recebe continuamente a informação de força. O trabalho faz parte do escopo do projeto "Teleoperação Avançada de Equipamentos de Mineração", que tem como objetivo o desenvolvimento de um arcabouço computacional que possibilite a teleoperação de modo mais eficaz, eficiente e seguro, utilizando técnicas de visão ativa, reconstrução tridimensional, interfaces hápticas, visualização de dados e planejamento. A Figura 1 ilustra o sistema em desenvolvimento.

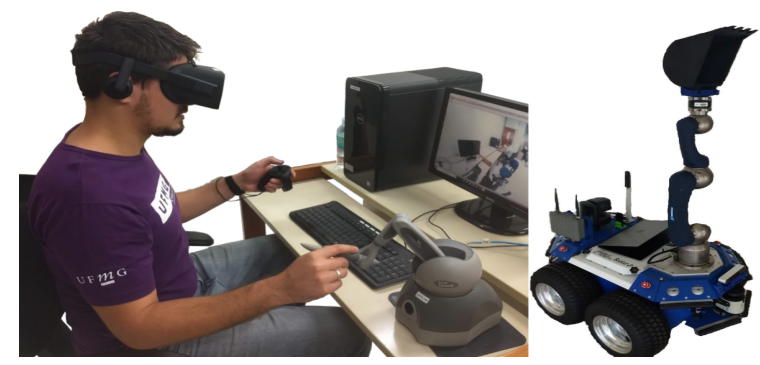

Figura 1. Arcabouço computacional em desenvolvimento para a teleoperação de escavadeiras.

Simulações foram feitas com o objetivo de avaliar a influência da realimentação do estresse sofrido pelo manipulador, e também na sensação de imersão provida ao operador. No sistema desenvolvido foi utilizado o Robot Operating System (ROS). O ROS é uma estrutura de código aberto para o desenvolvimento em robótica, suportando um modelo de troca de mensagens através da publicação e inscrição em tópicos (Quigley et al., 2009).

O restante deste trabalho está organizado da seguinte forma: a Seção 2 apresenta a modelagem cinemática e dinâmica do problema. A Seção 3 detalha a lei de controle utilizada e o mapeamento adotado entre os espaços de trabalho do dispositivo háptico e do manipulador. A Seção 4 descreve como ocorre a realimentação de força. A Seção 5 apresenta as simulações realizadas. Por fim, a Seção 6 resume as contribuições deste trabalho e descreve trabalhos futuros da pesquisa.

\section{MODELAGEM}

Uma escavadeira é comumente composta das seguintes partes: 1) base, 2) estrutura superior incluindo a cabine, e 3) manipulador fixado na região frontal. Considerase o manipulador da escavadeira uma entidade à parte que possui a configuração com lança de profundidade (Boom), lança de levante (Stick) e caçamba (Chacko et al., 2014). A Figura 2 ilustra tais elementos. O manipulador da escavadeira pode ser modelado como um mecanismo planar de três graus de liberdade com juntas de revolução (RRR).

Neste artigo, é utilizado o manipulador Schunk LWA $4 P$ para emular o funcionamento do braço de uma escavadeira, e a realimentação de força é implementada por um dispositivo háptico Geomagic Touch.

\subsection{Cinemática Direta}

O Schunk $L W A 4 P$ é um braço robótico antropomórfico com punho esférico totalizando seis graus de liberdade. O manipulador possui três graus de liberdade a mais do que 


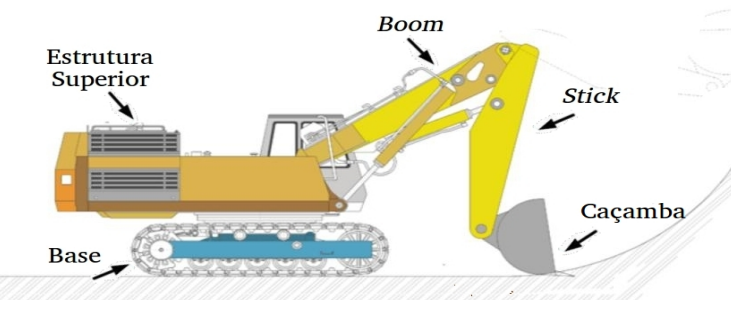

Figura 2. Ilustração das partes de uma escavadeira.

o braço de uma escavadeira, por isso pode ser considerado redundante.

O posicionamento dos sistemas de coordenadas dos elos foi definido pela representação de Denavit-Haterberg (DH). A Tabela 1 foi adaptada de (Memar e Esfahani, 2015), onde os parâmetros DH padrões do manipulador Schunk $L W A 4 P$ emulando uma escavadeira são especificados. A Figura 3 apresenta os sistemas de coordenadas dos elos do manipulador.

Tabela 1. Parâmetros DH - Schunk LWA4P.

\begin{tabular}{ccccc} 
Elo & $\theta_{i}$ & $d_{i}$ & $a_{i}$ & $\alpha_{i}$ \\
\hline 1 & $\theta_{1}$ & $0.1 \mathrm{~m}$ & 0 & $\pi / 2$ \\
2 & $\theta_{2}+\pi / 2$ & 0 & $0.350 \mathrm{~m}$ & $\pi$ \\
3 & $\theta_{3}+\pi / 2$ & 0 & 0 & $\pi / 2$ \\
4 & $\theta_{4}$ & $0.305 \mathrm{~m}$ & 0 & $-\pi / 2$ \\
5 & $\theta_{5}$ & 0 & 0 & $\pi / 2$ \\
6 & $\theta_{6}$ & $0.09 \mathrm{~m}$ & 0 & 0 \\
\hline
\end{tabular}

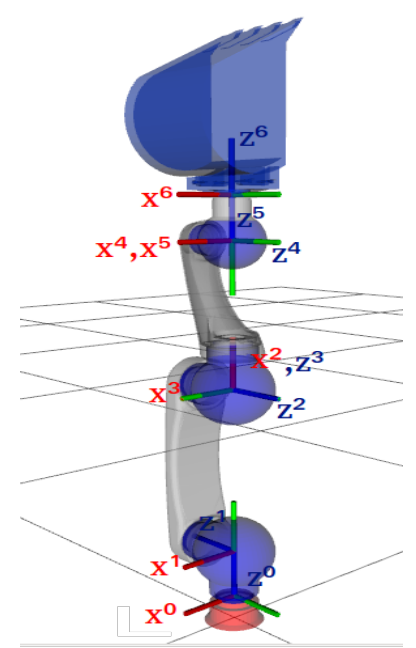

Figura 3. Sistemas de coordenadas do manipulador Schunk LWA 4 P.

O Geomagic Touch é um dispositivo háptico profissional que oferece seis graus de liberdade. Três de suas juntas são atuadas, o que o permite aplicar forças à mão do usuário. Além disso, o dispositivo possibilita um alto grau de flexibilidade com um design portátil por meio de uma caneta removível e dois botões integrados.

Os parâmetros de DH também são utilizados para a modelagem cinemática direta do dispositivo háptico Geomagic Touch. Os parâmetros DH padrões estão especificados na Tabela 2. A Figura 4 ilustra os sistemas de coordenadas dos elos do dispositivo háptico.
Tabela 2. Parâmetros DH - Geomagic Touch

\begin{tabular}{ccccc} 
Elo & $\theta_{i}$ & $d_{i}$ & $a_{i}$ & $\alpha_{i}$ \\
\hline 1 & $\theta_{1}$ & $0.125 \mathrm{~m}$ & 0.0 & $\pi / 2$ \\
2 & $\theta_{2}$ & 0.0 & $0.14 \mathrm{~m}$ & 0 \\
3 & $\theta_{3}$ & 0 & 0 & $\pi / 2$ \\
4 & $\theta_{4}$ & $0.1325 \mathrm{~m}$ & 0 & $-\pi / 2$ \\
5 & $\theta_{5}+\pi / 2$ & 0 & 0 & $-\pi / 2$ \\
6 & $\theta_{6}$ & $-0.07 \mathrm{~m}$ & 0 & 0 \\
\hline
\end{tabular}

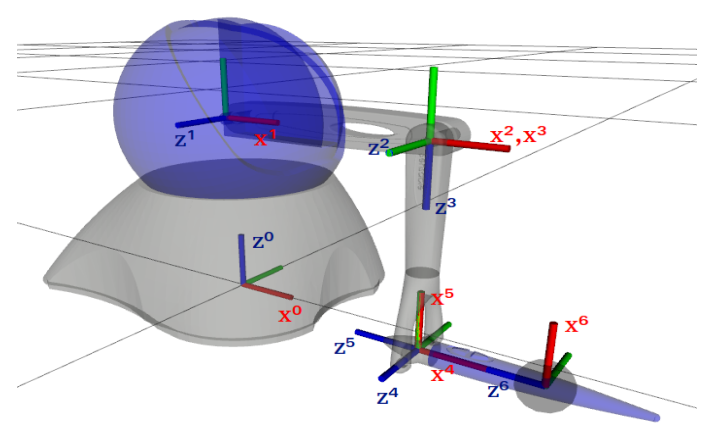

Figura 4. Sistemas de coordenadas do dispositivo háptico Geomagic Touch.

Uma forma simples da aplicação desses modelos no ambiente ROS é a utilização de arquivos Unified Robot Description Format (URDF), que descrevem o modelo do robô em linguagem Extensible Markup Language (XML). Os URDF's utilizados nesse trabalho foram adaptados para os parâmetros apresentados aqui a partir de (IPA, 2018) e (HumaRobotics, 2018).

\subsection{Cinemática Diferencial}

O problema da cinemática diferencial de um manipulador serial consiste em, dado as posições e velocidades das juntas, descobrir a velocidade do efetuador. A relação entre as velocidades das juntas $(\dot{\theta})$ e as velocidades lineares $v$ e angulares $\omega$ do efetuador $\left(\dot{X} \triangleq\left[\begin{array}{llllll}v_{x} & v_{y} & v_{z} & \omega_{x} & \omega_{y} & \omega_{z}\end{array}\right]^{T}\right)$ no espaço da tarefa é dada por uma matriz denominada Jacobiana geométrica $(J(\theta))$, que depende da configuração $(\theta)$ do manipulador (Spong et al., 2006). Essa relação é expressa por $(1)$ :

$$
\dot{X}=\frac{\partial X}{\partial \theta} \dot{\theta}=J(\theta) \dot{\theta} .
$$

A Jacobiana pode ser dividida em duas partes, uma em relação às velocidades lineares $\left(J_{p} \in \mathbb{R}^{3 \times n}\right)$ e outra em relação as velocidades angulares $\left(J_{o} \in \mathbb{R}^{3 \times n}\right)$, i.e., $J(\theta)=$ $\left[J_{p}^{T} J_{o}^{T}\right]^{T}$.

Ao longo deste artigo será necessário realizar operações que envolvem obter a inversa da matriz Jacobiana, que nem sempre é quadrada, impossibilitando a inversão direta desta matriz. A generalização da inversa para uma matriz não-quadrada é dada pela matriz pseudo-inversa, porém esta pode ser computada somente quando a matriz Jacobiana possuir posto pleno, o que não é satisfeito em configurações singulares. Para contornar este problema é utilizada a inversa Damped Least-Squares (DLS) proposta por (Nakamura e Hanafusa, 1986). A inversa DLS é definida como: 


$$
J^{\#}=J^{T}\left(J J^{T}+\lambda I\right)^{-1},
$$

onde $\lambda$ é um fator de amortecimento que leva a um melhor condicionamento do ponto de vista numérico da matriz. O valor de $\lambda$ é calculado como:

$$
\lambda=\left\{\begin{array}{l}
0 \\
\lambda_{0}\left(1-\frac{w}{w_{0}}\right)^{2}, \text { se } w \geq w_{0} \\
\text { se } w<w_{0}
\end{array},\right.
$$

onde $w=\sqrt{\operatorname{det} J(\theta) J^{T}(\theta)}$ é a manipulabilidade do sistema, $\lambda_{0}$ define o comportamento da solução na vizinhança de uma singularidade, e $w_{0}$ define os limites dessa vizinhança.

\subsection{Dinâmica - Torque gravitacional}

O conhecimento do torque gravitacional se faz necessário para o cálculo da força aplicada ao efetuador quando esse não possui um sensor de força instalado. No caso de manipuladores com elos rígidos, a única fonte de energia potencial do sistema é a gravidade.

O vetor de carga gravitacional $g(\theta)$ pode ser obtido a partir da energia potencial como:

$$
g(\theta)=\frac{\partial U}{\partial \theta}
$$

A energia potencial $(U)$ do manipulador, por sua vez, é obtida a partir das contribuições de cada elo $\left(U_{i}\right)$ e pode ser computada assumindo que a massa de todo o elo está concentrada em seu centro de massa, tal que:

$$
U=\sum_{i=1}^{n} U_{i}=\sum_{i=1}^{n} g_{0}^{T} r_{i} m_{i}
$$

sendo o vetor de aceleração gravitacional $g_{0}^{T}=\left[\begin{array}{lll}0 & 0 & -9,81\end{array}\right]$ $\mathrm{m} / \mathrm{s}^{2}, r_{i}$ a posição do centro de massa em relação ao sistema de coordenadas da base e $m_{i}$ a massa do elo.

Em um ambiente simulado é fácil determinar as forças gravitacionais uma vez que o próprio usuário pode definir os parâmetros como distâncias entre elos, massas e centros de massa. No ambiente real, no entanto, esses parâmetros nem sempre estão disponíveis ou não são simples de se obter. Uma solução nessas situações é fazer a identificação dinâmica do sistema como proposto em (Memar e Esfahani, 2015).

\section{CONTROLE CINEMÁTICO}

Para que o braço da "escavadeira"se posicione de maneira desejada é utilizada a lei de controle cinemático baseado na Matriz de Transformação Homogênea (MTH) (Caccavale et al., 1999). O objetivo de controle é tal que:

$$
\lim _{t \rightarrow \infty} H=H_{d}
$$

onde $H=\left[\begin{array}{ll}R & p \\ \mathbf{0} & 1\end{array}\right]$ é a MTH atual do manipulador e $H_{d}=\left[\begin{array}{cc}R_{d} & p_{d} \\ \mathbf{0} & 1\end{array}\right]$ é a MTH desejada, ambas pertencem ao grupo especial euclidiano de ordem $3(S E(3))$. As matrizes $R$ e $R_{d}$ são matrizes de rotação pertencentes ao grupo de rotação $(S O(3))$ e $p, p_{d} \in \mathbb{R}^{3}$ são vetores de posição tridimensional.
Inicialmente, é necessário definir os erros de posição e de orientação, formalizados como:

$$
e_{p}=p_{d}-p
$$

e

$$
\tilde{R}=R_{d} R^{T}
$$

respectivamente.

Seguindo a formulação proposta em (Caccavale et al., 1999), o erro de orientação é reescrito considerando o mapeamento de matriz de rotação para a representação dada pelo par eixo-ângulo $\tilde{R} \mapsto \phi \boldsymbol{n}$, onde $\boldsymbol{n} \in \mathbb{R}^{3}$ é o eixo de rotação e $\phi \in \mathbb{R}$ é o ângulo de rotação associados com $\tilde{R}$.

A entrada de controle é dada por

$$
u=J^{\#} K\left[\begin{array}{c}
e_{p} \\
\phi \boldsymbol{n}
\end{array}\right],
$$

onde $K \in \mathbb{R}^{6 \times 6}$ é a matriz de ganho definida positiva. $\mathrm{O}$ vetor $u \in \mathbb{R}^{6}$ define as entradas de controle para cada junta, dadas como velocidades angulares das juntas:

$$
u_{i}=\dot{\theta}_{i}, \quad(i=1, \cdots, 6),
$$

onde $\dot{\theta}_{i}$ é a velocidade angular da $i$-ésima junta, $u_{i}$ é o sinal de controle de velocidade aplicado ao driver do atuador da $i$-ésima junta. A inversa DLS $\left(J^{\#} \in \mathbb{R}^{6 \times 6}\right)$ apresentada na Seção 2 é utilizada para fazer o mapeamento entre o espaço da tarefa e o espaço das juntas. A Figura 5 ilustra o esquema de controle proposto.

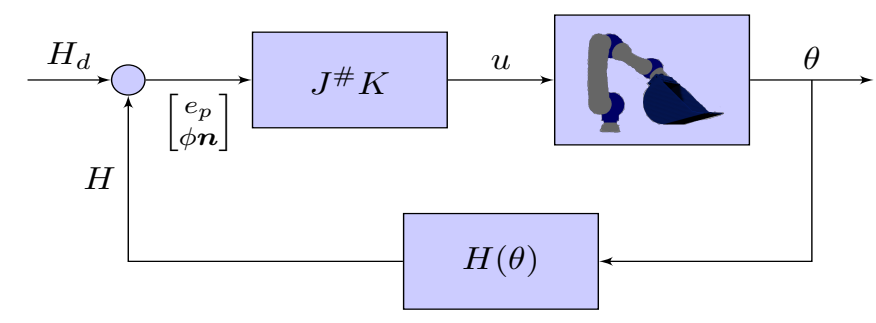

Figura 5. Esquema do controlador cinemático proposto.

\subsection{Mapeamento do espaço de trabalho}

O dispositivo háptico é utilizado pelo operador para definir a pose de referência do manipulador. A definição das posições e orientações desejadas são realizadas separadamente. Para obter a posição desejada é necessário calcular a cinemática direta e fazer o mapeamento entre os espaços de trabalho do dispositivo háptico e do manipulador. Já para obter a orientação, foi utilizada uma estratégia onde a cinemática direta é calculada desconsiderando algumas das juntas.

O espaço de trabalho de um dispositivo háptico é geralmente diferente do espaço de trabalho de um robô manipulador. Por esse motivo, a posição desejada $p_{d}$ não pode ser simplesmente a posição atual do mestre. Desta forma, é necessário fazer um mapeamento entre os espaços de trabalho do mestre e do escravo. A forma mais simples deste mapeamento é utilizar um fator de escala na posição do dispositivo háptico. Ao adotar um valor grande como fator de escala, o manipulador é capaz de realizar grandes deslocamentos a partir de pequenos movimentos do dispositivo háptico; o que traz como desvantagem a maior 
dificuldade de se realizar movimentos que requerem maior precisão.

Uma solução é utilizar a abordagem balística, na qual a perda de resolução espacial, quando elevados valores são escolhidos no mapeamento proporcional, é solucionada ao considerar a velocidade do deslocamento do dispositivo háptico (Conti e Khatib, 2005). Quando o dispositivo háptico é movido rapidamente, assume-se que um posicionamento grosseiro é desejado, então um alto valor do fator de escala é utilizado. Já quando o dispositivo é movido lentamente, assume-se que uma maior precisão é desejada, utilizado assim um menor valor para o fator de escala (Chotiprayanakul e Liu, 2009). Assim, é possível obter um controle preciso ao se realizar movimentos grosseiros, possibilitando, também a realização de movimentos mais finos.

Considerando a aplicação proposta, além do mapeamento dos espaços de trabalho, também é necessário adicionar uma restrição à posição desejada definida por $p_{d} \triangleq$ $\left[\begin{array}{lll}p_{d_{x}} & p_{d_{y}} & p_{d_{z}}\end{array}\right]^{T}$. Como o braço da escavadeira está restrito ao plano $x z$, a componente $p_{d_{y}}$ deve ser constante e igual a zero.

A posição desejada $p_{d}$ é obtida por:

$$
p_{d}=\left[\begin{array}{lll}
p_{d_{y}}^{\prime} & 0 & p_{d_{z}}^{\prime}
\end{array}\right]^{T},
$$

onde

$$
\left[p_{d_{x}}^{\prime} p_{d_{y}}^{\prime} p_{d_{z}}^{\prime}\right]^{T}=p_{d_{o l d}} k\left(\dot{p}_{h}\right)\left(p_{h}-p_{h_{o l d}}\right)
$$

sendo $p_{h}$ a posição da caneta do dispositivo háptico com respeito ao sistema de coordenada de sua base, obtida via cinemática direta considerando todas as juntas do dispositivo háptico $\theta_{D H}=\left[\begin{array}{lll}\theta_{D H_{1}} & \cdots & \theta_{D H_{6}}\end{array}\right] \cdot \dot{p}_{h}$ é a velocidade da caneta do dispositivo háptico, $p_{d_{\text {old }}}$ a posição desejada anterior e $p_{h_{\text {old }}}$ a posição anterior do dispositivo háptico.

A função de escala $k\left(\dot{p}_{h}\right)$ é definida como:

$$
k\left(\dot{p}_{h}\right)=\left\{\begin{array}{ll}
k_{f}\left\|\dot{p}_{h}\right\| & , \text { se }\left\|\dot{p}_{h}\right\|>v_{\text {lim }} \\
k_{s} & , \text { se }\left\|\dot{p}_{h}\right\| \leq v_{\text {lim }}
\end{array},\right.
$$

onde $k_{f}$ e $k_{s}$ são constantes positivas e $v_{l i m}$ é o limiar da velocidade da ponta do dispositivo háptico.

\subsection{Seleção da orientação desejada}

O movimento do braço da escavadeira deve ser restrito ao plano $x z$ do sistema de coordenadas da base. O dispositivo háptico por sua vez não é restringido, o que torna difícil definir diretamente uma orientação a ser transmitida para o manipulador.

Como solução, para orientar a caçamba, a cinemática direta do dispositivo háptico é calculada novamente, mas agora considerando apenas o movimento da junta 5 , ou seja, o vetor de posições angulares do dispositivo é dado por $\theta_{D H}^{*}=\left[\begin{array}{llllll}0 & 0 & 0 & 0 & \theta_{D H_{5}} & 0\end{array}\right]$. Desta forma, a matriz de rotação $R_{d}$ representa uma rotação elementar em torno do eixo $y$ do sistema de coordenadas da base, satisfazendo a restrição planar.

\section{REALIMENTAÇÃO DE FORÇA}

Na busca por uma melhor sensação de imersão e melhorias no desempenho na realização de tarefas, muitos sistemas mestre-escravo incorporam a realimentação de força. Nesse caso, o operador é capaz de sentir as forças que estão sendo aplicadas ao escravo devido à interação com o ambiente remoto (Niemeyer et al., 2016).

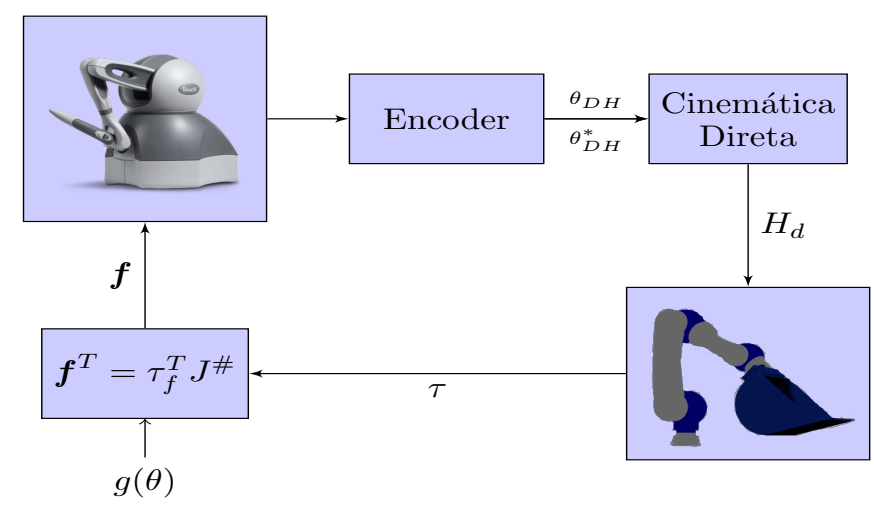

Figura 6. Diagrama de realimentação de força.

Como apresentado em (Siciliano et al., 2010), a análise estática de um manipulador estabelece uma relação entre as forças e torques aplicados ao efetuador e forças/torques aplicadas às juntas. Por meio do princípio do trabalho virtual, essa relação é descrita por:

$$
\tau_{f}=J_{p}^{T} \boldsymbol{f}
$$

sendo $\tau_{f} \in \mathbb{R}^{6 \times 1}$ o vetor dos torques resultantes nas juntas devido às forças externas, $J_{p} \in \mathbb{R}^{3 \times 6}$ a matriz Jacobiana geométrica de posição e $\boldsymbol{f} \in \mathbb{R}^{3 \times 1}$ o vetor de forças aplicadas no efetuador do manipulador.

É usual em (14) o uso da Jacobiana geométrica e também o uso do vetor de forças e torques $\left(F=\left[\boldsymbol{f}^{T} \boldsymbol{n}^{T}\right]^{T}\right)$. Devido ao fato de que o dispositivo háptico empregado possui somente as três primeiras juntas atuadas, optou-se por ignorar os torques aplicados no efetuador.

A Equação (14) somente é válida para o equilíbrio estático, mas apresenta uma boa aproximação para quando a condição de equilíbrio não é satisfeita.

Na aplicação proposta, a variável a ser calculada é a força aplicada no efetuador, que deve ser explicitada isolando o termo $\boldsymbol{f}$ no lado esquerdo da equação.

Além disso, com o manipulador em repouso, o vetor de torques $\tau$ aplicados nas juntas pode ser visto como a soma dos torques devido a aplicação da força externa $\tau_{f}$ com os torques gravitacionais $g(\theta)$, tal que $\tau=\tau_{f}+g(\theta)$.

Utilizando a inversa DLS de (2), a Equação (14) pode ser reescrita como:

$$
\begin{aligned}
\tau_{f} & =J(\theta)^{T} \boldsymbol{f} \\
\tau_{f}^{T} & =\boldsymbol{f}^{T} J(\theta) \\
\boldsymbol{f}^{T} J(\theta) J^{\#} & =\tau_{f}^{T} J^{\#} \\
\boldsymbol{f}^{T} & \approx(\tau-g(\theta))^{T} J^{\#} .
\end{aligned}
$$

A Equação (15) é utilizada para estimar a força aplicada no efetuador do manipulador da escavadeira que, em seguida, será transmitida ao operador por meio da interface háptica. A Figura 6 ilustra o esquema da realimentação. A força computada no espaço operacional deve ser mapeada para torque no espaço das juntas. Tipicamente isto é feito 
utilizando (14), mas agora empregando a jacobiana do dispositivo háptico. A exata força desejada é sentida pelo operador caso o dispositivo não tenha dinâmica, não esteja próximo de uma configuração singular e o atuador seja ideal. Entretanto, a dinâmica real, os atrasos no tempo, entre outros aspectos não ideais, podem influenciar na força aplicada e consequentemente na percepção do operador (Niemeyer et al., 2016).

\section{SIMULAÇÕES}

A arcabouço utilizado para avaliação experimental é composto pelo dispositivo háptico Geomagic Touch conectado via Ethernet a um computador rodando ROS Kinetic no Ubuntu 16.04. O manipulador Schunk LWA $4 P$ foi simulado na plataforma Gazebo 7, que possui integração com o ROS.

O experimento consiste em utilizar o dispositivo háptico a fim de teleoperar o manipulador para realizar a tarefa de transferir algumas esferas entre duas caixas, de forma similar à uma operação de carregamento de minério. A Figura 7(a) apresenta o ambiente virtual onde a tarefa é realizada.

São avaliadas duas situações: 1) sistema sem realimentação de força e 2) sistema com realimentação de força. O objetivo é comparar o nível de estresse (força externa aplicada sobre manipulador) ao qual o manipulador é submetido durante a execução da tarefa. A força de interação é medida por um sensor de força localizado na junta 6 do manipulador.

As esferas possuem os seguintes parâmetros: raio $0,05 \mathrm{~m}$ e massa de $0,25 \mathrm{~kg}$. A matriz utilizada de ganho $K$ da lei de controle em (9) é $K=5 I_{6}$, sendo $I_{6}$ a matriz identidade de ordem 6. No cálculo da inversa DLS em (3), foram considerados os seguinte valores: $w_{0}=0,001$ e $\lambda_{0}=0,5$. Os parâmetros utilizados na abordagem balística em (13) são: $k_{f}=3,0, k_{s}=1,2$ e $v_{l i m}=0,4 \mathrm{~m} / \mathrm{s}$.

Durante a execução da tarefa, é possível observar o estado corrente do manipulador por meio de uma animação na interface visual. A fim de aproximar o ambiente simulado das condições da operação de uma escavadeira real, o campo de visão virtual do usuário foi limitado de forma similar ao que um operador de escavadeira possui. As Figuras 7(b)-7(c) ilustram, respectivamente, o campo de visão nos ambientes simulado e real.

O ângulo de visão do operador pode não ser adequado para identificar a existência e a quantidade de material dentro da caçamba. Além disso, em ambientes como minas à céu aberto é comum o aparecimento de cortinas de poeira, o que também é prejudicial à realimentação visual. Desta forma, a realimentação de força possui importante papel em determinar se a caçamba está vazia ou carregada.

Foram realizadas 10 rodadas de simulações, metade com o sistema de realimentação de força desligado e a outra metade com a realimentação ativada. Todas as simulações foram realizadas pelo mesmo usuário. Cada rodada teve duração de 40 segundos.

Foi possível observar durante as simulações que quando o usuário não tem a realimentação de força, não é possível distinguir entre situações onde a caçamba está carregada ou não. Quando isto ocorre, a etapa de carregamento da caçamba é repetida antes que ocorra o descarregamento do material na outra caixa. Isto não ocorre nas simulações com realimentação, pois o usuário tem noção do peso do material dentro da caçamba.

A Tabela 3 apresenta as forças máximas, as médias e as integrais das forças captadas pelo sensor de força durante as simulações sem realimentação das forças de interação. A Tabela 4 mostra as mesmas informações quando o operador tem realimentação de força.

\begin{tabular}{cccc}
$\mathrm{N}$ & Máximo $F_{s}(N)$ & Média de $F_{s}(N)$ & $\int_{0}^{40} F_{s} d t$ \\
\hline 1 & 81,8 & 12,0 & 481,2 \\
2 & 64,6 & 11,1 & 443,9 \\
3 & 104,2 & 10,6 & 423,1 \\
4 & 66,1 & 10,4 & 417,3 \\
5 & 83,1 & 10,0 & 400,8 \\
\hline Média & 80,0 & 10,8 & 433,3
\end{tabular}

Tabela 3. Intensidade da força aplicada à caçamba - Sistema sem realimentação de força ao usuário.

\begin{tabular}{cccc}
$\mathrm{N}$ & Máximo $F_{c}(N)$ & Média de $F_{c}(N)$ & $\int_{0}^{40} F_{c} d t$ \\
\hline 1 & 56,9 & 9,7 & 387,7 \\
2 & 76,4 & 9,1 & 364,8 \\
3 & 64,3 & 8,7 & 347,5 \\
4 & 58,1 & 7,9 & 316,0 \\
5 & 36,8 & 8,0 & 278,4 \\
\hline Média & 58,5 & 338,9 \\
Tabela & 4. Intensidade da força aplicada à ca- \\
çamba - Sistema com realimentação de força & ao usuário.
\end{tabular}

É possível concluir das tabelas que quando o operador não possui a realimentação, ele empurra o manipulador contra os objetos do ambiente causando forças de reação em média 36\% maiores. Esse aumento do estresse do manipulador tem impactos negativos como a redução de sua vida útil.

Outra forma de comparar os efeitos da realimentação de força é por meio dos gráficos nas Figuras 8-12. Cada gráfico mostra a evolução da intensidade da força de interação nas duas situações: uma simulação sem realimentação e outra com realimentação. Os gráficos combinam as simulações de acordo com as Tabelas 3-4, que estão em ordem decrescente do valor da integral das forças.

Infere-se das Figuras 8-12 que, sem realimentação de força, é comum o usuário comandar o manipulador de forma a gerar maiores forças de interação com o ambiente. Isto é devido ao fato de que somente com a realimentação visual, o usuário não percebe e forma clara quando o manipulador já está em contato com objetos rígidos localizados no ambiente remoto.

\section{CONCLUSÃO}

Esse artigo apresentou um arcabouço para a teleoperação de escavadeiras com a realimentação das forças de interação com ambiente para o usuário.

As simulações demonstraram que a realimentação de força serve como complemento ao realimentação visual. Quando não é possível identificar visualmente se a caçamba está 


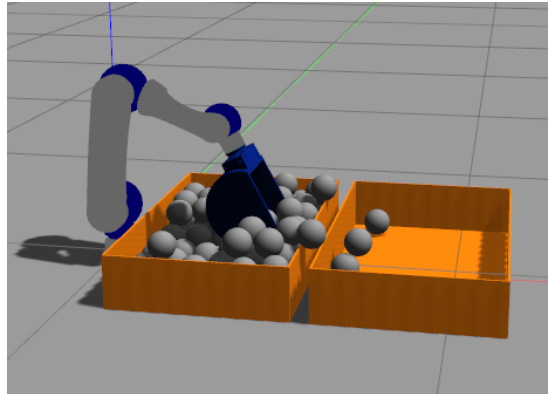

(a) Vista lateral da execução da tarefa.

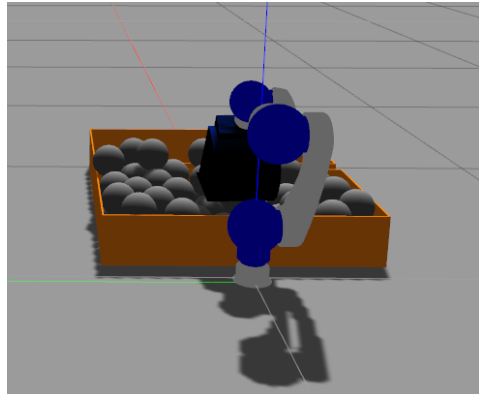

(b) Campo de visão do usuário ao executar a tarefa em ambiente simulado.

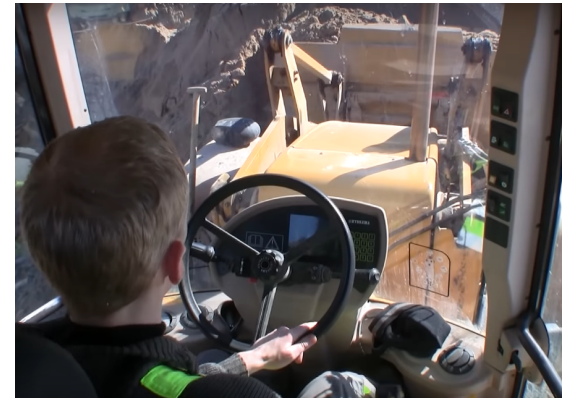

(c) Campo de visão do usuário ao executar a tarefa em ambiente real.

Figura 7. Diferentes perspectivas do ambiente virtual utilizado nas simulações, e comparação com a visão de um operador de escavadeiras.

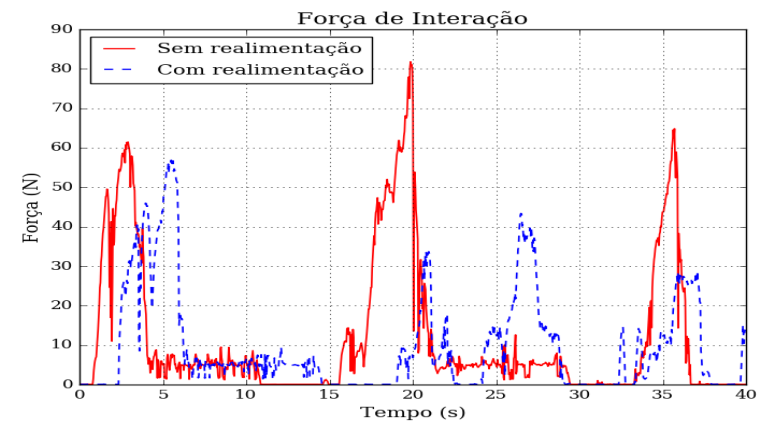

Figura 8. Gráfico do módulo das forças aplicadas ao efetuador durante a execução da tarefa $\left(\int_{0}^{40} F_{s} d t=481,2\right.$ e $\left.\int_{0}^{40} F_{c} d t=387,7\right)$.

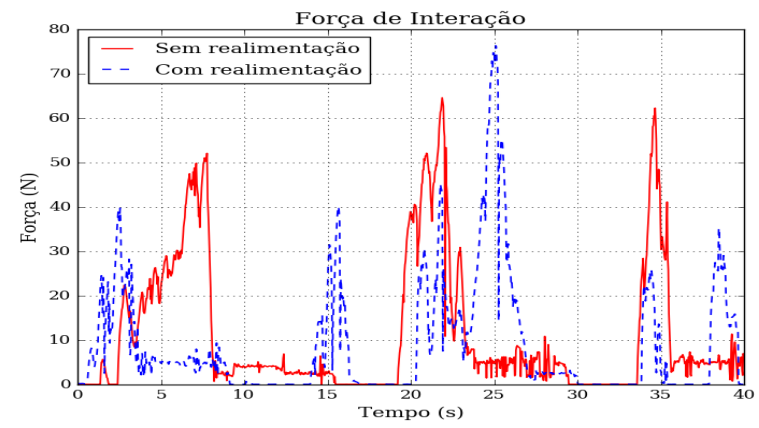

Figura 9. Gráfico do módulo das forças aplicadas ao efetuador durante a execução da tarefa $\left(\int_{0}^{40} F_{s} d t=443,9\right.$ e $\left.\int_{0}^{40} F_{c} d t=364,8\right)$.

carregada, o usuário, por meio da interface háptica, consegue ter boa noção do material manipulado.

Outra vantagem do sistema de realimentação de força é que o manipulador sofre menos estresse; durante as simulações, foi verificado que a utilização do sistema acarretou numa redução média de $36 \%$ das forças aplicadas ao manipulador. O operador, ao deslocar o manipulador contra objetos rígidos, sente em suas mãos a força de reação e desta forma evita contatos que podem danificar o manipulador. Caso não houvesse a realimentação de força, o usuário poderia continuar forçando o manipulador contra o ambiente, causando forças de interação excessivas.

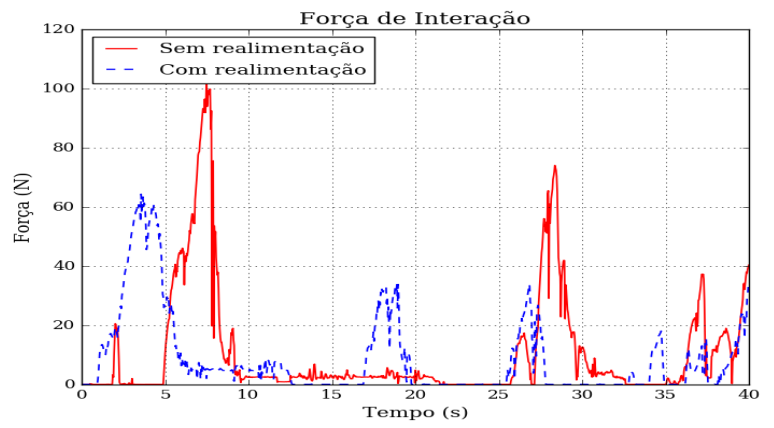

Figura 10. Gráfico do módulo das forças aplicadas ao efetuador durante a execução da tarefa $\left(\int_{0}^{40} F_{s} d t=423,1\right.$ e $\left.\int_{0}^{40} F_{c} d t=347,5\right)$.

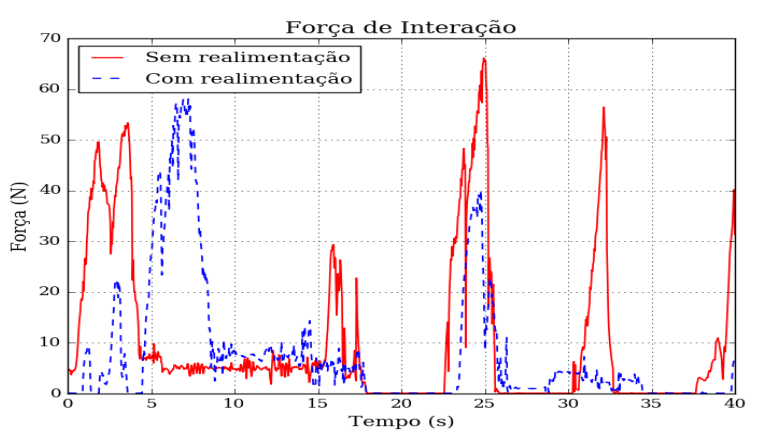

Figura 11. Gráfico do módulo das forças aplicadas ao efetuador durante a execução da tarefa $\left(\int_{0}^{40} F_{s} d t=417,3\right.$ e $\left.\int_{0}^{40} F_{c} d t=316,0\right)$.

Em pesquisas futuras este arcabouço será implementado em um manipulador real. A estratégia de realimentação de força também será integrada e utilizada no sistema de teleoperação descrito em (Rezeck et al., 2018), permitindo maior imersão ao usuário através de outros instrumentos de realimentação visual e/ou auditiva como óculos de realidade virtual.

\section{REFERÊNCIAS}

Ahn, K. (2004). Development of force reflecting joystick for hydraulic excavator. JSME International Journal 


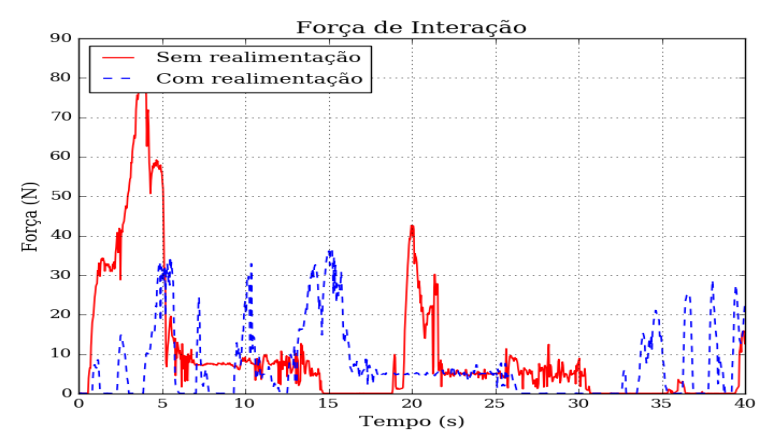

Figura 12. Gráfico do módulo das forças aplicadas ao efetuador durante a execução da tarefa $\left(\int_{0}^{40} F_{s} d t=400,8\right.$ e $\left.\int_{0}^{40} F_{c} d t=278,4\right)$.

Series C Mechanical Systems, Machine Elements and Manufacturing, 47(3), 858-863.

Caccavale, F., Siciliano, B., e Villani, L. (1999). The role of euler parameters in robot control. Asian journal of control, 1(1), 25-34.

Chacko, V., Yu, H., Cang, S., e Vladareanu, L. (2014). State of the art in excavators. In Proceedings of the 2014 International Conference on Advanced Mechatronic Systems, 481-488. IEEE.

Chotiprayanakul, P. e Liu, D. (2009). Workspace mapping and force control for small haptic device based robot teleoperation. In 2009 International Conference on Information and Automation, 1613-1618. IEEE.

Conti, F. e Khatib, O. (2005). Spanning large workspaces using small haptic devices. In First Joint Eurohaptics Conference and Symposium on Haptic Interfaces for Virtual Environment and Teleoperator Systems. World Haptics Conference, 183-188. IEEE.

Hokayem, P.F. e Spong, M.W. (2006). Bilateral teleoperation: An historical survey. Automatica, 42(12), 20352057.

HumaRobotics (2018). Geomagic touch description. URL https://github.com/HumaRobotics/geomagic_ touch. [Online; acessado em 02-Abril-2019].

IPA, F. (2018). Schunk robots description. URL https://github.com/ipa320/schunk_modular_ robotics/tree/indigo_dev/schunk_description. [Online; acessado em 02-Abril-2019].

Lichiardopol, S. (2007). A survey on teleoperation. Technische Universitat Eindhoven, DCT report.

Memar, A.H. e Esfahani, E.T. (2015). Modeling and dynamic parameter identification of the schunk powerball robotic arm. In ASME 2015 International Design Engineering Technical Conferences and Computers and Information in Engineering Conference, V05CT08A024V05CT08A024. American Society of Mechanical Engineers.

Nakamura, Y. e Hanafusa, H. (1986). Inverse kinematic solutions with singularity robustness for robot manipulator control. Journal of dynamic systems, measurement, and control, 108(3), 163-171.

Niemeyer, G., Preusche, C., Stramigioli, S., e Lee, D. (2016). Telerobotics. In Springer handbook of robotics, 1085-1108. Springer.

Okamura, A.M. (2009). Haptic feedback in robot-assisted minimally invasive surgery. Current opinion in urology,
19(1), 102.

Park, J. e Khatib, O. (2006). A haptic teleoperation approach based on contact force control. The International Journal of Robotics Research, 25(5-6), 575-591. doi: 10.1177/0278364906065385.

Quigley, M., Conley, K., Gerkey, B., Faust, J., Foote, T., Leibs, J., Wheeler, R., e Ng, A.Y. (2009). Ros: an opensource robot operating system. In ICRA workshop on open source software, volume 3, 5. Kobe, Japan.

Rezeck, P., Cadar, F., Soares, J., Frade, B., Pinto, L., Azpurua, H., Macharet, D.G., Chaimowicz, L., Freitas, G., e Campos, M.F.M. (2018). An immersion enhancing robotic head-like device for teleoperation. In 2018 Latin American Robotic Symposium, 2018 Brazilian Symposium on Robotics (SBR) and 2018 Workshop on Robotics in Education (WRE). IEEE. doi:10.1109/lars/ sbr/wre.2018.00038. URL https://doi.org/10.1109/ lars/sbr/wre.2018.00038.

Roberts, J.M., Duff, E.S., e Corke, P.I. (2002). Reactive navigation and opportunistic localization for autonomous underground mining vehicles. Information Sciences, 145(1-2), 127-146.

Siciliano, B., Sciavicco, L., Villani, L., e Oriolo, G. (2010). Robotics: modelling, planning and control. Springer Science \& Business Media.

Spong, M.W., Hutchinson, S., Vidyasagar, M., et al. (2006). Robot modeling and control. John Wiley \& Sons. 